Original Article

\title{
Histological dermal changes caused by preparation and application procedures in percutaneous dose toxicity studies in dogs, rabbits and rats
}

\author{
Mikio Mitsuishi ${ }^{1}$, Takafumi Oshikata ${ }^{1}$, Shino Kumabe ${ }^{2}$, Azusa Kobayashi ${ }^{1}$, Koshiro Katoku ${ }^{1}$, \\ Takeshi Kanno ${ }^{1}$, Masao Hamamura ${ }^{1}$, and Minoru Tsuchitani ${ }^{2 *}$ \\ ${ }^{1}$ Kumamoto Laboratory, Nonclinical Research Center, LSI Medience Corporation, 1285 Kurisaki-machi, Uto-shi, Kumamoto \\ 869-0425, Japan \\ ${ }^{2}$ Kashima Laboratory, Nonclinical Research Center, LSI Medience Corporation, 14-1 Sunayama, Kamisu-shi, Ibaraki 314-0255, \\ Japan
}

\begin{abstract}
We reevaluated histological slides of dorsal skin in control animals from past percutaneous dose toxicity studies using dogs, rabbits and rats to provide background data concerning histological changes related to preparation and application procedures and vehicles or embrocations of every variety. Acanthosis, dermal or perifollicular inflammatory cell infiltration in dogs; hyperkeratosis, acanthosis, dermal inflammatory cell infiltration or hemorrhage in rabbits; and acanthosis, dermal inflammatory cell infiltration, crust or foreign body granuloma in rats were present as procedure-related underlying histological changes in the control animals. Four mechanical acts, (1) rubbing with gauze to remove an administered substance for reapplication, (2) use of a taut bandage to avoid slipping from the application site, (3) peeling a patch off as a preparation procedure for reapplication, and (4) clipping or shaving, were considered to cause injury to the skin. The degree of influence of the various application procedures was found to be as follows: sham, lotion $<$ cream < ointment and tape in dogs; untreated control, sham < lotion $<$ tape and poultice in rabbits; and sham, sodium carboxymethylcellulose < olive oil and lotion < ointment and tape in rats. The degree of ointment influence on rabbits is equivocal. (DOI: 10.1293/ tox.2014-0021; J Toxicol Pathol 2015; 28: 1-9)
\end{abstract}

Key words: percutaneous dose toxicity, acanthosis, dermal toxicity, dog, rabbit, rat

\section{Introduction}

In recent years, the forms of formulations of existing drugs have shifted to external preparations, as percutaneous administration of drugs has many advantages such as few adverse effects and convenience for medication and maintenance of blood concentrations ${ }^{1,2}$. As a part of the toxicological evaluation of pharmaceutical agents (external preparations) and cosmetics, repeated dose toxicity studies by percutaneous administration including cumulative skin irritation studies in rabbits are conducted to investigate general or local toxicity ${ }^{3}$. In skin irritancy studies, it is well known that inflammation may be seen focally in controls where skin abrasion techniques have been employed ${ }^{3}$. In

Received: 28 April 2014, Accepted: 7 July 2014

Published online in J-STAGE: 8 August 2014

*Corresponding author: M Tsuchitani

(e-mail Tsuchitani.Minoru@mp.medience.co.jp)

(C)2015 The Japanese Society of Toxicologic Pathology

This is an open-access article distributed under the terms of the Creative Commons Attribution Non-Commercial No Derivatives (by-ncnd) License $<$ http://creativecommons.org/licenses/by-nc-nd/3.0/> . nonclinical safety studies of developing pharmaceuticals, a few reports described the presence of cellular infiltration or squamous cell hyperplasia in the skin of control animals that have received percutaneous application of vehicle ${ }^{4,5}$, while some reports described the absence of lesions in the skin of controls ${ }^{6-9}$. For precise evaluation of test article-related changes, it is important to accurately perceive underlying changes caused by dermal administration procedures and vehicles by referring to background histopathological data of sham-treated and vehicle control animals from various percutaneous dose toxicity studies. However, there are currently no reports dealing with the influences of the preparation and application procedures and vehicles or embrocations of every variety. We reevaluated histological slides of dorsal skin from past percutaneous dose toxicity studies in dogs, rabbits and rats to clarify possible histological changes in sham-treated and vehicle control animals and evaluated the degree of the lesions caused by various embrocations.

\section{Materials and Methods}

We reevaluated archived histological slides from 15 toxicity studies in beagle dogs (four 2-week studies, six 
4-week studies and five 13-week studies), 15 skin irritation studies in rabbits (4-week study) and 11 toxicity studies in rats (4-week study) conducted at the Kumamoto Laboratory of the Nonclinical Research Center of LSI Medience Corporation.

\section{Animals in the original studies}

Dogs: Beagle dogs of both sexes were purchased from Covance Research Products Inc., Cumberland, VA, USA, at the age of 5 or 6 months for use in 12 studies, and beagle dogs of both sexes were acquired from Kitayama Labes Co., Ltd., Yamaguchi, Japan, at the age of 6, 8 or 9 months for use in 3 studies.

Rabbits: Male New Zealand White rabbits (Kbs:NZW) were used in 12 studies, female New Zealand White rabbits (Kbs:NZW) were used in 1 study, and male Japan White rabbits (Kbl:JW) were used in 2 studies; all rabbits were purchased from Kitayama Labes Co., Ltd., Nagano, Japan, at 3 or 4 months.

Rats: $\mathrm{Crl}: \mathrm{CD}(\mathrm{SD})$ rats of both sexes were purchased from Charles River Laboratories Japan, Inc., Shiga, Japan, at 4 weeks for use in 11 studies.

\section{Preparation and application procedures in the origi- nal studies}

The preparation and application procedures used for each species are described below and summarized in Table 1.

Dogs: As the preparation procedure, the hair on the dorsal skin was removed with electric clippers (Speedik DC-6, blade $0.1 \mathrm{~mm}$, Shimizu Electric Co., Ltd., Osaka, Japan) and an electric shaver (BS 5442, Braun, Kronberg, Germany) before application, and hair removal was performed 2 to 3 times a week. The application areas for all dogs excluding those applied tape were covered with a sheet of lint cloth or a double gauze cloth and bound with surgical tape (Micropore, 3M Health Care). These areas were covered with a sheet of lint cloth and bound with an expansible and contractile bandage (ELASTOPORE, Nichiban, Tokyo, Japan) again. The application areas for dogs applied tape were covered with a sheet of lint cloth and bound with an expansible and contractile bandage once. After these treatments, all dogs were fitted with a jacket and an Elizabethan collar during the administration period. After exposure for 20 to 24 hours daily, the gauze cloth, lint cloth and jacket were removed. The skin of the applied area of all dogs except for the tape treatment animals was wiped with a gauze sheet immersed in lukewarm water and with dry tissue paper and then dried with a hair drier (cool blast). On the other hand, the tape was peeled off from the dogs in the tape group for reapplication. These procedures were then repeated.

Rabbits: The hair on the dorsal skin was removed with electric clippers once a week. No skin abrasion technique was employed in this study. The application areas for rabbits applied lotion or ointment were left open during the administration period. Saline or water for injection was applied to the sham treatment animals, the application area of which was also left open. Similarly, the dorsal area of the untreated
Table 1. Summary of the Preparation and Application Procedures in Each Group for Each Species

\begin{tabular}{llccccc}
\hline \multirow{2}{*}{ Group } & \multicolumn{2}{c}{ Hair } & Applica- Gauze/ & Jacket & Collar \\
& Sham treatment & Yes & No & Yes & Yes & Yes \\
& Lotion & Yes & Yes & Yes & Yes & Yes \\
\multirow{4}{*}{ Dog } & Cream & Yes & Yes & Yes & Yes & Yes \\
& Ointment & Yes & Yes & Yes & Yes & Yes \\
& Tape & Yes & Yes & Yes & Yes & Yes \\
\hline \multirow{6}{*}{ Rabbit } & Untreated control & Yes & No & No & No & Yes \\
& Sham treatment & Yes & S/W & No & No & Yes \\
& Ootion & Yes & Yes & No & No & Yes \\
& Ointment & Yes & Yes & No & No & Yes \\
& Tape & Yes & Yes & Yes & No & Yes \\
& Poultice & Yes & Yes & Yes & No & Yes \\
\hline \multirow{6}{*}{ Rat } & Sham treatment & Yes & No & Yes & No & Yes \\
& CMC-Na & Yes & Yes & Yes & No & Yes \\
& Olive oil & Yes & Yes & Yes & No & Yes \\
& Lotion & Yes & Yes & Yes & No & Yes \\
& Ointment & Yes & Yes & Yes & No & Yes \\
& Tape & Yes & Yes & Yes & No & Yes \\
\hline
\end{tabular}

a) S/W: Applied saline or water for injection.

controls was left open. The application areas of the rabbits applied tape or poultice were covered with absorbent cotton, a nonwoven adhesion bandage (MESHPORE, Nichiban, Tokyo, Japan) and an expansible and contractile bandage. After treatment, all rabbits were fitted with an Elizabethan collar during the administration period. The application area was cleaned daily in a manner similar to that in the dogs described above.

Rats: Removal of the hair on the dorsal skin with electric clippers and an electric shaver was performed on a schedule that would avoid hair growth (every second day to daily). The application areas of all rats including the sham treatment animals were covered with a sheet of lint cloth twice or an impermeable sheet over a gauze cloth and bound with an expansible and contractile bandage. After treatment, all rats were fitted with an Elizabethan collar during the administration period. The application area was cleaned daily in a manner similar to that in the dogs described above.

\section{Embrocations and patches}

Lotion, cream, ointment and tape were applied to the dorsal skin of dogs; lotion, ointment, tape and poultice were applied to the dorsal skin of rabbits; and sodium carboxymethylcellulose (CMC-Na), olive oil, lotion, ointment and tape were applied to the dorsal skin of rats, as shown in Table 1. The saline or water for injection was applied to the rabbits in the sham treatment group.

In all the original studies, the animals were cared for according to the principles outlined in the guides for the care and use of laboratory animals prepared by the Japanese Association for Laboratory Animal Science and our institution. In addition, at the commencement of these studies, the study protocols were reviewed and approved by the Institutional Animal Care and Use Committee of LSI Medience Corporation. 


\section{Histopathology}

In all studies, the dorsal skin was fixed in $10 \%$ neutral buffered formalin, embedded in paraffin, cut into $5-\mu \mathrm{m}-$ thick sections and stained with hematoxylin and eosin. All histological slides were reevaluated and graded according to the following criteria.

Acanthosis (common to dogs, rabbits and rats): "minimal", over twice the thickness of the epidermis in the untreated normal skin; "mild", over three times thickness to that of the epidermis in the untreated normal skin, and the enlarged nucleus of keratinocyte to be oval; "moderate", over five times thickness to that of the epidermis in the untreated normal skin, and clearly defined three layers of basal, spinous and granular

Inflammatory cell infiltration, dermis (common to dogs, rabbits and rats): "minimal", one to two foci composed of ten to twenty inflammatory cells, or a few scattered inflammatory cells limited to the superficial dermis just underneath the epidermis; "mild", rather large foci of inflammatory cells, over three times the size of the minimal lesion, or diffuse inflammatory cell infiltration in the upper half area of dermis underneath the epidermis; "moderate", thick and diffuse inflammatory cell infiltration spreading to deep dermis

Inflammatory cell infiltration, perifollicle (present in dogs only): "minimal", a few scattered inflammatory cells in the perifollicular area; "mild", thick inflammatory cells localized in the perifollicular area; "moderate", thick and widespread inflammatory cell infiltration encircling the follicle

Hemorrhage, dermis (present in rabbits only): "minimal", one to two minute hemorrhagic foci; "mild", over four focal hemorrhages; "moderate", widespread multi-focal or diffuse hemorrhages

In the rabbits, the area of island skin was excluded from the evaluation specimens. In the rats, the thickness of the epidermis was evaluated taking into account variations due to the hair growth cycle.

To numerically compare the degree of each lesion between groups, the minimal, mild and moderate grades were transcribed into scores of 1, 2 and 3, respectively. The group sum and mean were calculated.

\section{Results}

\section{Histopathological findings}

The histopathological findings in the dogs, rabbits and rats are summarized in Tables 2-4.

Dogs: Acanthosis, inflammatory cell infiltration in the dermis and perifollicular inflammatory cell infiltration were detected in the animals applied cream, ointment and tape (Fig. 1). The inflammatory cells included mainly neutrophils in dogs (Fig. 1F, G, I, J). Acanthosis frequently coincided with hyperplasia of the follicular epithelium (Fig. 1D). The grade and incidence of these lesions in the ointment and tape groups showed a tendency to increase as compared with the cream group (Table 2). Moderate acanthosis was detected in the animals applied ointment or tape in the 13week study only (Fig. 1D). Acanthosis was also detected in the sham treatment animals, although most of them showed minimal lesions. No significant change was detected in the animals applied lotion. A single lesion of folliculitis was found in a sham treatment animal as an incidental finding.

Rabbits: Acanthosis and inflammatory cell infiltration in the dermis were detected in the animals applied lotion, ointment, tape and poultice (Fig. 2). The inflammatory cells were composed of lymphocytes and pseudoeosinophils in various proportions in rabbits (Fig. $2 \mathrm{~F}-\mathrm{H}$ ). The grade and incidence of acanthosis and inflammatory cell infiltration in the poultice group showed a tendency to increase, and the grade of acanthosis in the tape group also showed a tendency to increase, as compared with the lotion group (Table 3 ). Moderate changes in acanthosis and inflammatory cell infiltration were detected only in the poultice group (Fig. $2 \mathrm{D}, \mathrm{H})$. In the sham treatment animals applied saline or water for injection, inflammatory cell infiltration in the dermis was detected in two of 18 rabbits only, and one of the two also had acanthosis. Hemorrhage in the dermis was detected in five animals applied tape and one animal applied poultice (Fig. 2I-K). Hyperkeratosis could not be graded and was present in six animals applied lotion and two animals applied poultice. No significant change was noticed in the untreated control animals.

Rats: Acanthosis and inflammatory cell infiltration in the dermis were detected in all groups except for the CMC$\mathrm{Na}$ group, in which acanthosis was not present (Fig. 3). The inflammatory cells mainly included lymphocyte in rats (Fig. $3 \mathrm{H}, \mathrm{I})$. Moreover, animals in which lymphocytes infiltrated exclusively were included. However, neutrophils were prominent in some animals. The grade and incidence of acanthosis in the ointment and tape groups showed a tendency to increase as compared with the sham treatment, olive oil and lotion groups (Table 4). The grade and incidence of acanthosis in females was higher than that in males in the sham treatment, olive oil, lotion, ointment and tape groups. The grade and/or incidence of inflammatory cell infiltration in the dermis in the olive oil, lotion, ointment and tape groups showed a tendency to increase as compared with the sham treatment and CMC-Na groups. Crust could not be graded but was present in a few animals applied $\mathrm{CMC}-\mathrm{Na}$, lotion and ointment. Also, foreign body granuloma could not be graded but was present in the deep dermis or upper subcutis of a few sham treatment animals and a few animals applied CMC-Na, olive oil and ointment. The granuloma was composed of an accumulation of histiocytes and multinucleated giant cells containing hair fragments in the center (Fig. 3J). A single lesion, a dermal cyst was found as an incidental finding in one animal applied CMC-Na.

\section{Score of each lesion}

The mean scores of each finding were calculated for each group, and the scores are shown in a bar graph in Fig. 4. In dogs, the scores in the ointment and tape groups of both sexes in all the 2-, 4-, 13-week studies were clearly 


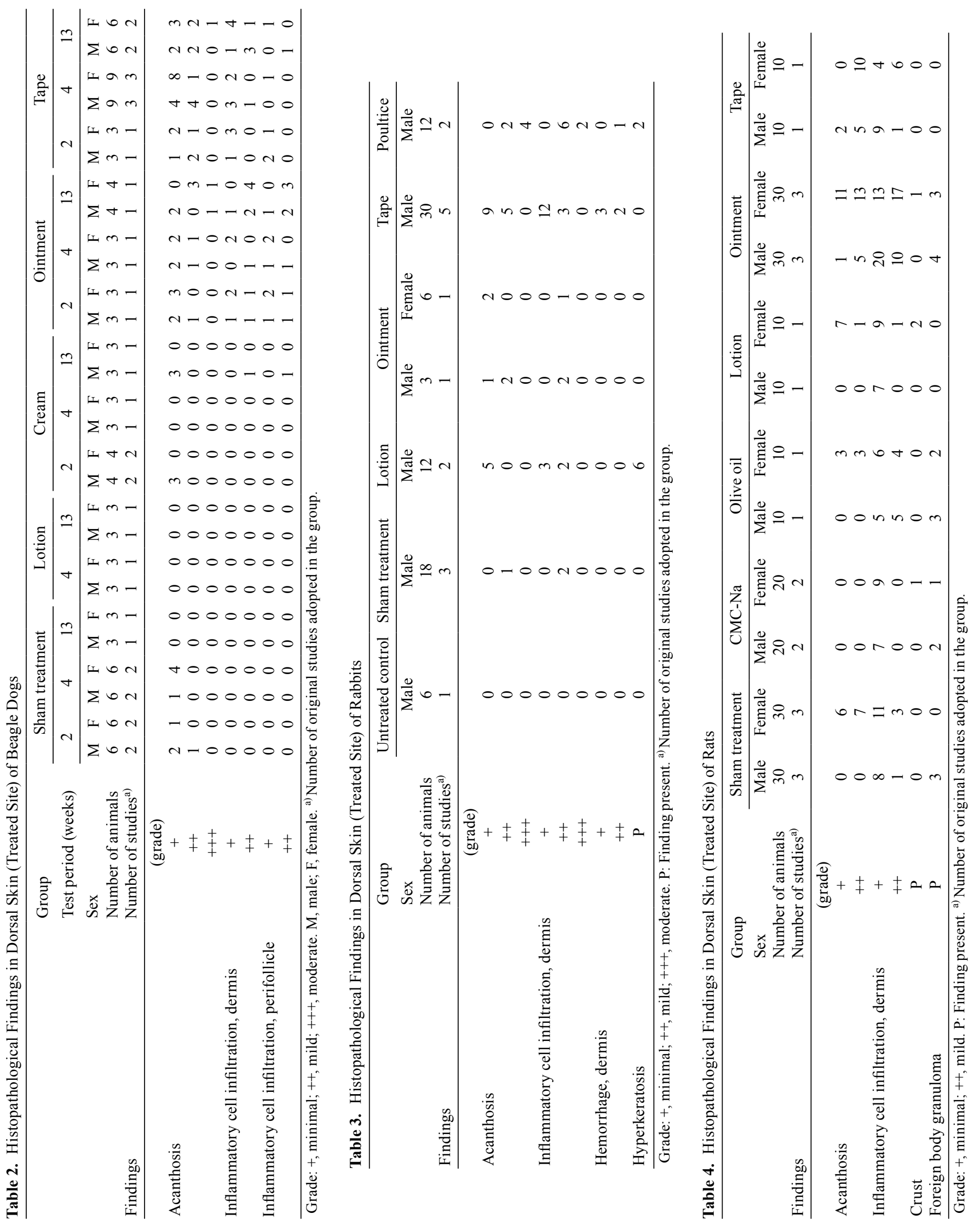



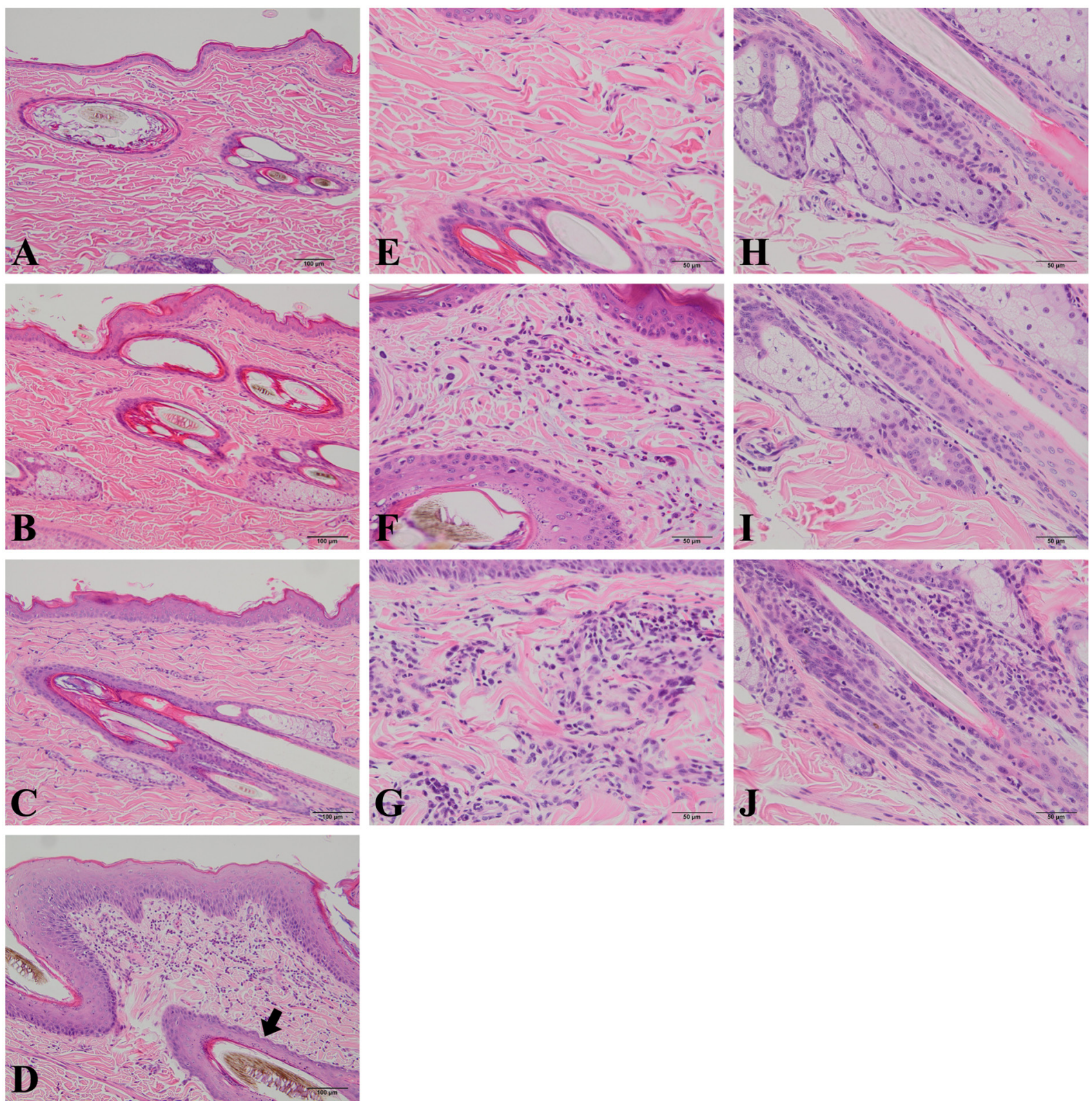

Fig. 1. Photomicrographs showing the histopathology of the skin in dogs. A: Normal appearance of the epithelium in the dog of the sham group. Bar $=100 \mu \mathrm{m}$. B, C and D: Minimal (B), mild (C) and moderate (D) acanthosis of the dogs applied cream, tape and tape, respectively. The follicular epithelium is also hyperplastic (arrow). Bar $=100 \mu \mathrm{m}$. E: Normal appearance of the dermis in the dog of the sham group. Bar $=50 \mu \mathrm{m}$. F, G: Minimal (F) and mild (G) inflammatory cell infiltration in the dermis of the dogs applied ointment. The neutrophils are prominent. Bar $=50 \mu \mathrm{m} . \mathrm{H}$ : Normal appearance of the perifollicular area in the dog of the sham group. Bar $=50 \mu \mathrm{m}$. I, J: Minimal (I) and mild (J) perifollicular inflammatory cell infiltration of the dogs applied ointment. Bar $=50 \mu \mathrm{m}$.

higher than those in the sham treatment groups (Fig. 4A). Moreover, the score in the ointment group was higher than that in the tape group because of a higher score for perifollicular inflammatory cell infiltration in the former. The score of the male dogs in the cream group of the 13-week study was also higher. In rabbits, the scores of the males in the ointment and poultice groups were clearly higher than that of the untreated control or sham treatment group (Fig. 4B). The scores of the males in the lotion and tape groups were the next highest after the two groups stated above.
In rats, the scores of both sexes in the ointment and tape groups were clearly higher than that of the sham treatment group (Fig. 4C). The olive oil and lotion groups also showed higher scores than the sham treatment group in comparisons among the same sexes.

\section{Discussion}

In this study, it was confirmed that the preparation and application procedures employed in the percutaneous 

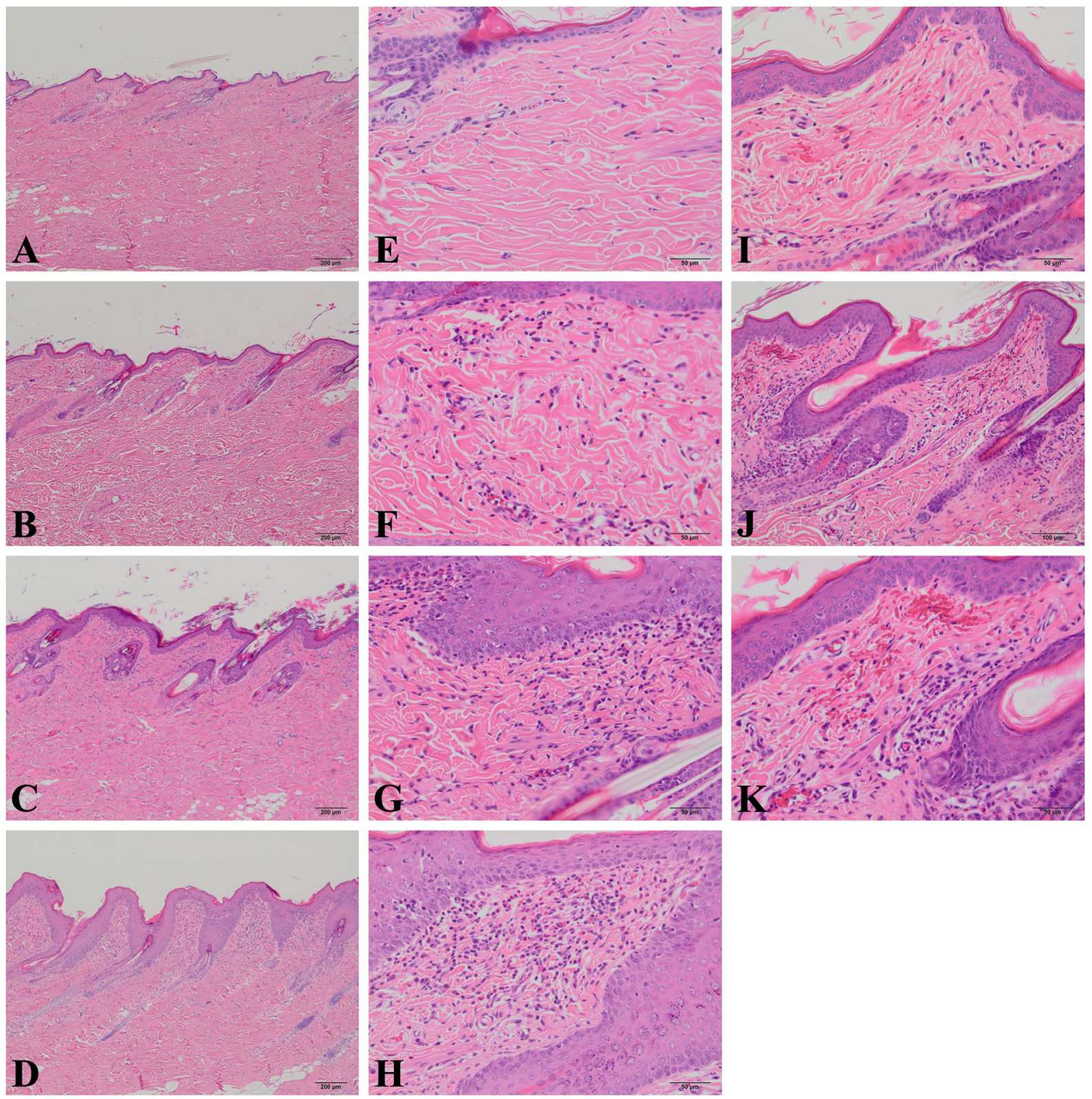

Fig. 2. Photomicrographs showing the histopathology of the skin in rabbits. A: Normal appearance of the epithelium in the rabbit of the sham group. Bar $=200 \mu \mathrm{m} . \mathrm{B}, \mathrm{C}$ and D: Minimal (B), mild (C) and moderate (D) acanthosis of the rabbits applied tape, poultice and poultice, respectively. Bar $=200 \mu \mathrm{m}$. E: Normal appearance of the dermis in the rabbit of the sham group. Bar $=50 \mu \mathrm{m} . \mathrm{F}, \mathrm{G}$ and H: Minimal (F), mild $(\mathrm{G})$ and moderate $(\mathrm{H})$ inflammatory cell infiltration in the dermis of the rabbits applied tape, poultice and poultice, respectively. The pseudoeosinophils are visible. Bar $=50 \mu \mathrm{m}$. I: Minimal hemorrhage in the dermis of the rabbit applied tape. Bar $=50 \mu \mathrm{m}$. J, K: Mild hemorrhage in the dermis of the rabbit applied tape. Photographs show the same area with low $(\mathrm{J}, \mathrm{Bar}=100 \mu \mathrm{m})$ and high $(\mathrm{K}, \mathrm{Bar}=50$ $\mu \mathrm{m})$ magnification.

dose toxicity studies induced acanthosis and inflammatory cell infiltration in the dermis as the main change common to dogs, rabbits and rats. Application of irritant substances causes a variety of histological changes, such as erosion or ulcer, and inflammation that ranges in severity ${ }^{3,10}$. However, when the intensity of the irritant is mild or moderate, the epidermis does not erode but instead shows reactive changes including hyperkeratosis and acanthosis ${ }^{3}$. In percutaneous dose toxicity studies, it is crucial to know the possible variations in histology of the preparation and application procedure-related lesions to discriminate test article-related changes from the underlying lesions occurring in the vehicle control group. The images and scores of the histopathological changes caused by the preparation and application procedures in this report represent valuable knowledge that will help in accurately determining the underlying lesions. Moreover, the scoring of each finding is useful for detection of the extent of lesions semiquantitatively ${ }^{3}$. 

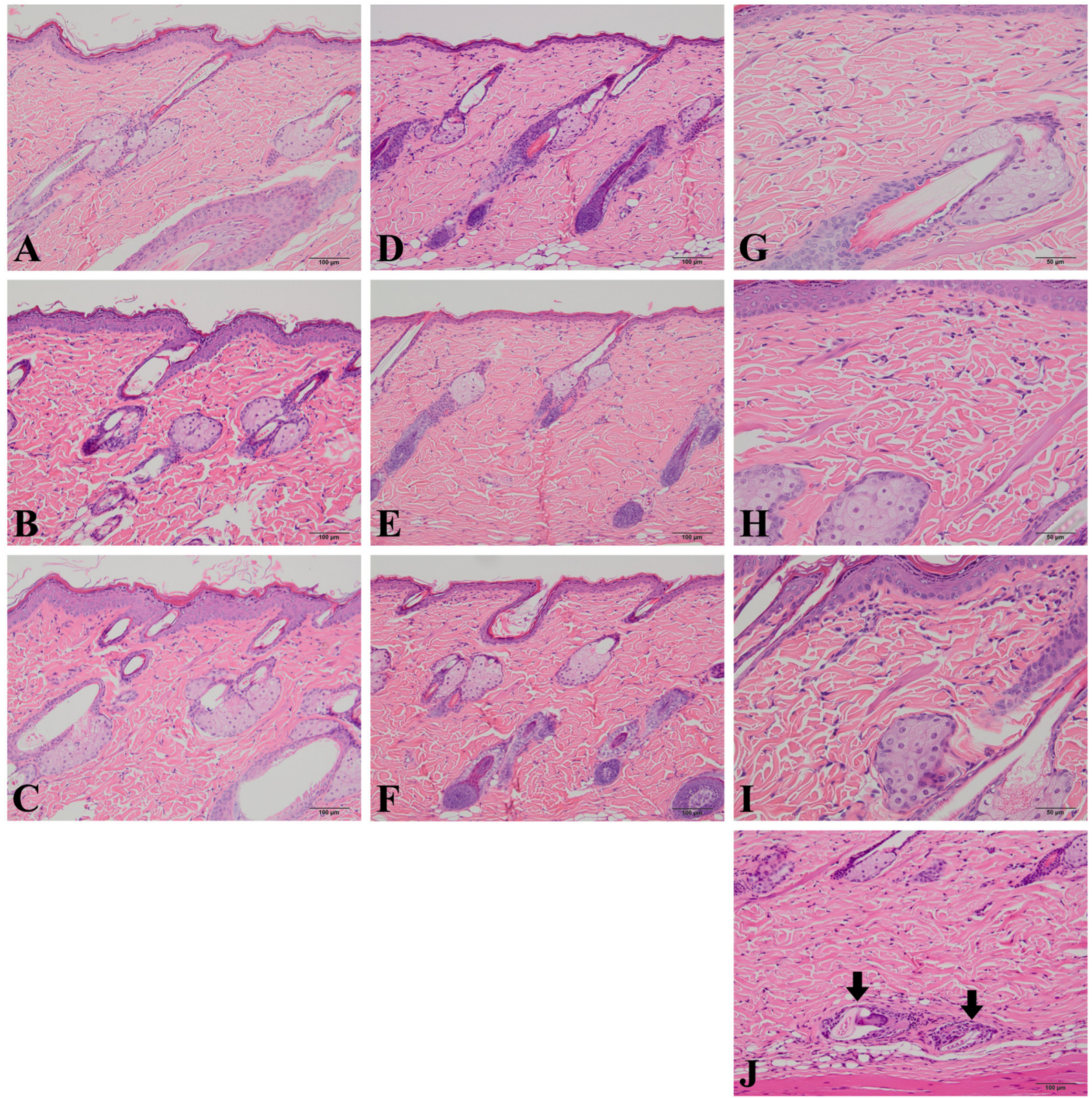

Fig. 3. Photomicrographs showing the histopathology of the skin in rats. A, D: Normal appearances of the epithelium in the male (A) and female (D) rats of the sham group. Bar $=100 \mu \mathrm{m}$. B, C: Minimal (B) and mild (C) acanthosis of the male rats applied tape. Bar $=100 \mu \mathrm{m}$. E, F: Minimal (E) and mild (F) acanthosis of the female rats of the sham and tape groups, respectively. Bar $=100 \mu \mathrm{m}$. G: Normal appearance of the dermis in the rat of the sham group. Bar $=50 \mu \mathrm{m}$. H, I: Minimal (H) and mild (I) inflammatory cell infiltration of the rats applied tape. Bar $=50 \mu \mathrm{m}$. J: Foreign body granuloma in the deep dermis of the rat applied ointment. Note the hair fragments (arrows) in the center of the granuloma. Bar $=100 \mu \mathrm{m}$.

Physical acts represent one of the exogenous factors that can potentially cause injury to the skin. For example, callosities occur in a localized area of the skin as a result of application of pressure or friction, and decubitus ulcers are the result of ischemic necrosis that follows application of a constant pressure ${ }^{11,12}$. Repeated clipping and shaving, occlusion over the skin by a sheet and bandage, repeated wiping with gauze and fitting of a jacket are considered to be possible factors that induced acanthosis and inflammatory cell infiltration in the present study, as none of the embrocations or patches contained any stimulating chemical substances. The score of the histopathological changes in the ointment group in the dogs, as well as those of the rabbits and rats, was clearly higher than that of the sham treatment group. The reason for this result was considered to be the stickiness of the ointment. Rubbing with gauze is required to wipe the administered substances off the skin when an embrocation is oily or sticky. Repeated rubbing can cause friction, a mechanical factor, to induce a dermal change ${ }^{11}$. Moreover, in the dog and rat cases, bandages can 

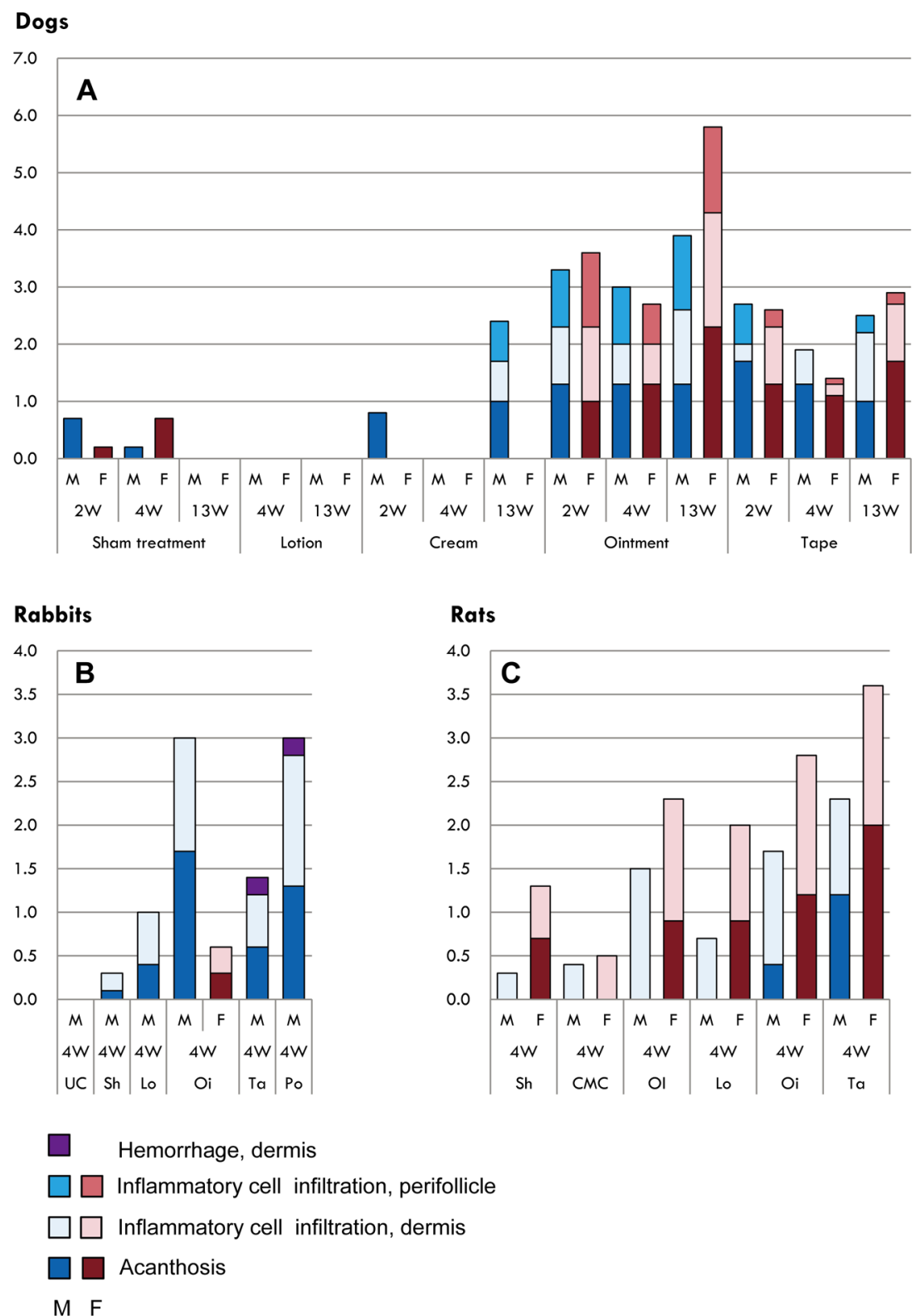

Fig. 4. Bar graphs showing the mean score of each finding in dogs (A), rabbits (B) and rats (C). The bars for the mean scores of each finding are stacked for each group. M, male; F, female; W, weeks; UC, untreated control; Sh, Sham treatment; Lo, lotion; Oi, ointment; Ta, tape; Po, poultice; $\mathrm{CMC}, \mathrm{CMC}-\mathrm{Na}$; Ol, olive oil.

put pressure on the skin if they are taut. To prevent bandages from slipping off the application site, bandages tend to be bound rather more tightly than usual if the embrocation is oily. Therefore, a taut bandage can be one of the mechanical factors that injure skin that has been clipped and shaved to remove the hair. The scores of the olive oil and ointment treatments being higher than that of the sham treatment in this study may reflect this supposition. Another possible mechanical cause is considered to be daily removal of the tape or poultice. Patch removal can cause various intensities of physical skin irritation due to various mechanical conditions such as removal speed, removal angle and application site temperature ${ }^{2}$. In this study, therefore, the higher scores in the tape and poultice groups may have been caused by the peeling action of patch removal. In the rabbit cases, we surmise that hemorrhage in the dermis was caused by patch removal because it was detected only in the tape and poultice groups.

Clipping or shaving can also be a factor that injures the skin, as lesions were present in the untreated control and sham treatment groups in the rabbit studies. The preparation procedure for the rabbits in these groups was merely repeated clipping and shaving. We suspect that the repeated clipping or shaving accidentally caused microscopic cuts on the surface of the skin.

Perifollicular inflammatory cell infiltration was de- 
tected in dogs, but not in rabbits and rats, in our study. No factors explaining this species difference were determined. However, we speculate that one possible factor that may be the cause of the species difference is the hair follicles of dogs being compound hair follicles, which have large common follicle orifices containing bundles of hairs (up to as many as fifteen hairs) ${ }^{13}$. Therefore, in the dog, it is possible that the vehicles penetrated well, stayed for a long period of time, and changed in quality with the help of its own secretion and indigenous bacteria in the follicle. Subsequently, the accumulated and changed substances may have influenced the follicular epithelium and perifollicle.

The score in the ointment group of male rabbits was much higher than that of the female rabbits. The reason for this difference is that the rabbits of both groups were adopted from different studies. The degree of stickiness or oiliness of the ointment may have been different between the male study and female study, as the ointments had different compositions in the different studies. Ultimately, the severity of the influence in the ointment group of rabbits was equivocal according to the results of our study.

In rats, the grade and incidence of acanthosis in females was higher than that in males in the sham treatment, olive oil, lotion, ointment and tape groups. The exact reason for this tendency is unknown. However, it may have been caused by the difference in vulnerability of the skin due to the epidermis of the females being basically thinner than that of the males, as the most critical defense against mechanical forces is the barrier derived from the superficial layers of the skin, which include the epidermis ${ }^{11}$.

In conclusion, we confirmed that preparation and application procedures induced histological dermal changes, principally acanthosis and inflammatory cell infiltration, in percutaneous dose toxicity studies. We also showed the features and grades of these lesions in each animal applied various embrocations and patches as background data for procedure-related lesions. The degree of influence of the various application procedures was found to be as follows: sham, lotion $<$ cream $<$ ointment and tape in dogs; untreated control, sham $<$ lotion $<$ tape and poultice in rabbits; and sham, CMC-Na $<$ olive oil and lotion $<$ ointment and tape in rats. The degree of ointment influence on rabbits is equivocal.

Acknowledgments: The authors would like to thank Mr. Stephen Filiatrault and Ms. Kanae Tamatsukuri for language editing. We have no financial relationships to disclose.

\section{References}

1. Kinoshita T, Akemi H, and Ohtsuka S. Preparation design and pharmaceutics basic knowledge of percutaneous ab- sorption pharmaceuticals. Shinryo to Shinyaku. 30: 166175. 1993; (in Japanese).

2. Kawamura N, Shinkai N, Yamauchi H, and Takayama S. Comparison of poultice-type and tape-type patches containing ketoprofen on human skin irritation. J Toxicol Sci. 28: 415-425. 2003. [Medline] [CrossRef]

3. Greaves P. Integumentary system. Skin and subcutaneous tissue. Non-neoplastic changes. In: Histopathology of Preclinical Toxicity Studies, 4th ed. P Greaves (ed). Elsevier, Amsterdam. 15-33. 2012.

4. Kitagaki T, Suzuki T, Koike Y, Ono M, Shirakawa K, Nagata M, and Konishi R. [A 4-week repeated percutaneous dose toxicity study of calcipotriol (MC903) followed by a 4-week recovery test in rats]. J Toxicol Sci. 21(Suppl 2): 287-307. 1996; (in Japanese). [Medline] [CrossRef]

5. Imaizumi T, Tsuruta M, Koike Y, Ono M, Shirakawa K, Nagata M, and Konishi R. [A 4-week repeated percutaneous dose toxicity study of calcipotriol (MC903) followed by a 4-week recovery test in dogs]. J Toxicol Sci. 21(Suppl 2): 309-323. 1996; (in Japanese). [Medline] [CrossRef]

6. Nagashima Y, Hisaoka F, Ide M, Tamura K, Shimura K, Tanaka G, and Tanaka H. [A 13-week percutaneous toxicity study of prednisolone farnesylate (PNF) gel in beagle dogs with a recovery period of 5 weeks]. J Toxicol Sci. 17(Suppl 3): 123-160. 1992; (in Japanese). [Medline] [CrossRef]

7. Nagashima $Y$, Ide M, Akai H, Tamura K, Shimura K, Tanaka H, and Tanaka G. [A 52-week percutaneous toxicity study of prednisolone farnesylate (PNF) gel in beagle dogs with a recovery period of 8 weeks]. J Toxicol Sci. 17(Suppl 3): 161-199. 1992; (in Japanese). [Medline] [CrossRef]

8. Kitagaki T, Suzuki T, Koike Y, Ono M, Shirakawa K, Nagata M, and Konishi R. [A 26-week repeated percutaneous dose toxicity study of calcipotriol (MC903) in rats]. J Toxicol Sci. 21(Suppl 2): 345-363. 1996; (in Japanese). [Medline] [CrossRef]

9. Imaizumi T, Tsuruta M, Koike Y, Ono M, Shirakawa K, Nagata M, and Konishi R. [A 26-week repeated percutaneous dose toxicity study of calcipotriol (MC903) in dogs]. J Toxicol Sci. 21(Suppl 2): 365-387. 1996; (in Japanese). [Medline] [CrossRef]

10. Klein-Szanto AJP, and Conti CJ. Skin and oral mucosa. In: Handbook of Toxicologic Pathology, 2nd ed. Volume 2. WM Haschek, CG Rousseaux, and MA Wallig (eds). Academic Press, San Diego. 85-119. 2002.

11. Hargis AM, and Ginn PE. The integument. In: Pathologic Basis of Veterinary Disease, 5th ed. JF Zachary, and MD McGavin (eds). Elsevier, St. Louis. 972-1084. 2012.

12. Ginn PE, Mansell JEKL, and Rakich PM. Skin and appendages. Physicochemical diseases of skin. In: Jubb, Kennedy, and Palmer's Pathology of Domestic Animals, 5th ed. Volume 1. M. Grant Maxie (ed). Elsevier, Edinburgh. 604-620. 2007.

13. Lovell JE, and Getty R. The sense organs and integument. The integument. In: Anatomy of The Dog, ME Miller, GC Christensen, and HE Evans (eds). W. B. Saunders Company, Philadelphia. 875-888. 1964. 